under $\mathrm{OA}$ conditions and is able to reduce the severity of cartilage destruction during $O A$.

Disclosure of Interest: None declared

DOI: 10.1136/annrheumdis-2017-eular.3689

\section{AB0032 UPREGULATION OF CD64 EXPRESSION ON MONOCYTES IN PATIENTS WITH ACTIVE ADULT-ONSET STILL'S DISEASE: A POSSIBLE BIOMARKER FOR ASSESSING DISEASE ACTIVITY}

E. Oguro $^{1}$, T. Shimizu ${ }^{1}$, A. Kikuchi-Taura ${ }^{2}$, S. Tsuji ${ }^{1}$, Y. Okita ${ }^{1}$, M. Shigesaka ${ }^{1}$, H. Matsuoka ${ }^{1}$, T. Nii ${ }^{1}$, S. Teshigawara ${ }^{1}$, E. Kudo-Tanaka ${ }^{1}$, Y. Harada ${ }^{1}$, M. Matsushita ${ }^{1}$, S. Ohshima ${ }^{2}$, Y. Saeki ${ }^{2} .{ }^{1}$ Department of Rheumatology and Allergology; ${ }^{2}$ Department of Clinical Research, NHO Osaka Minami Medical Center, Osaka, Japan

Background: Adult-onset Still's disease (AOSD) is a systemic inflammatory disease of unknown etiology. Overproduction of multiple inflammatory cytokines and subsequent hyperactivation of monocytes/macrophages are prominent characteristics of AOSD. However, there are no convenient and precise methods for evaluating monocyte/macrophage activation in AOSD. We previously reported that monocyte CD64 (mCD64) expression could be quantitatively measured by flow cytometry and its expression was tightly correlated with the activity of systemic lupus erythematosus.

Objectives: We examined the association between mCD64 expression and AOSD disease activity.

Methods: This was a prospective, single-center, observational study conducted between January 2013 and December 2016. Eleven active AOSD patients who fulfilled the Yamaguchi's criteria for AOSD and had the modified Pouchot score of $\geq 2$ were enrolled. The mCD64 expression levels were quantitatively measured by flow cytometry and individually assessed both before (Pouchot score $\geq 2$ ) and after treatment (score 0). Other disease-related laboratory data, such as C-reactive protein, ferritin, and white blood cell count, were simultaneously measured. As a control, 16 active systemic lupus erythematosus (SLE) patients (SLE disease activity index $\geq 6$ ), 22 active rheumatoid arthritis (RA) patients (disease activity score with 28-joint counts $>3.2$ ), and 20 healthy controls $(\mathrm{HC}$ ) (female, 55\%; mean age, $38.7 \pm 9.1$ years) were enrolled. Statistical analysis was performed by the Mann-Whitney and Wilcoxon-paired tests.

Results: The median mCD64 expression levels were 73,339 [interquartile range (IQR), 45,861-88,181] and 16,443 (IQR, 45,891-88,181) molecules/cell before and after treatment, respectively. Thus, mCD64 expression levels were significantly decreased during the inactive phase compared with those in the active phase in AOSD $(p=0.0001)$. The $m C D 64$ expression levels were significantly higher in patients with active AOSD than in those with active SLE [34,648 (IQR, 44,204-24,657) molecules/cell, $p=0.001$ ], active RA [25,167 (IQR, 35,77822,301) molecules/cell, $\mathrm{p}<0.0001]$, and in $\mathrm{HC}[14,174$ (IQR, 13,413-17,774) molecules/cell, $p<0.0001]$.

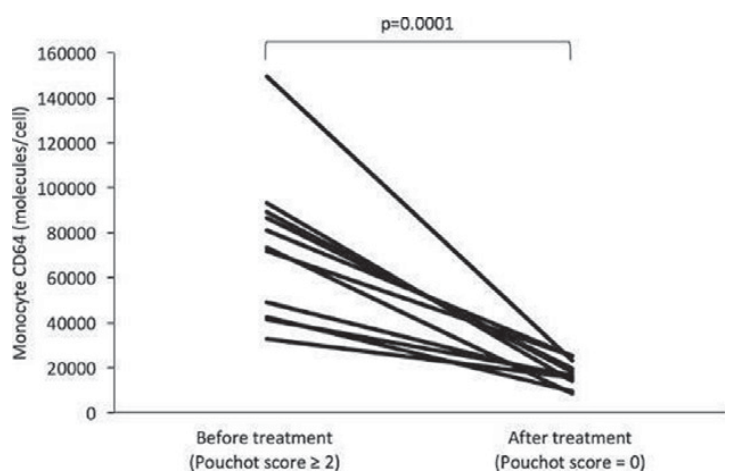

Conclusions: These results suggest that mCD64 expression levels are highly upregulated in AOSD and tightly correlated with disease activity. The mCD64 expression level may be a useful biomarker for assessing the disease activity of AOSD.

References:

[1] Rau M, et al. J Rheumatol. 2010 Nov;37(11):2369-76.

[2] Kikuchi-Taura A, et al. Lupus. 2015 Sep;24(10):1076-80.

[3] Pouchot J, et al. Medicine. 1991;70:118-36.

Disclosure of Interest: None declared

DOI: 10.1136/annrheumdis-2017-eular.3337

\section{AB0033 THE IDENTIFICATION OF IL-17A+, IL-17RA+ AND IL-17RC+ LYMPHOID AND MYELOID CELLS IN BLOOD OF TREATMENT NAIIVE EARLY AND IN SYNOVIAL FLUID OF ESTABLISHED PSORIATIC ARTHRITIS PATIENTS}

X. Xu ${ }^{1}$, N. Davelaar ${ }^{1}$, A.-M. Otten-Mus ${ }^{1}$, P.S. Asmawidjaja ${ }^{1}$, H. den Braanker ${ }^{1}$, H. Alves ${ }^{1}$, J.P. van Hamburg ${ }^{1}$, C. Gaillez ${ }^{2}$, J.M. Hazes ${ }^{3}$, R. Bisoendial ${ }^{4}$,

M. Vis ${ }^{3}$, F. Kolbinger ${ }^{5}$, E. Lubberts ${ }^{1} .{ }^{1}$ Rheumatology and Immunology, Erasmus
Mc, University Medical Center, Rotterdam, Netherlands; ${ }^{2}$ Novartis Pharma AG, Basel, Switzerland; ${ }^{3}$ Rheumatology, Erasmus Mc, University Medical Center; ${ }^{4}$ Rheumatology, Maasstad Hospital, Rotterdam, Netherlands; ${ }^{5}$ Novartis, Basel, Switzerland

Background: Interleukin (IL)-17A is a pro-inflammatory cytokine and is involved in the pathogenesis of psoriatic arthritis (PsA) $(1,2)$. Various cells can produce IL-17A. However, it is not clear which cell types in PsA patients are responsible for the production of IL-17A. In addition, the expression of IL-17RA and IL-17RC on different cell types is not well defined.

Objectives: To identify IL-17A, IL-17RA and IL-17RC positive cells in blood of first diagnosed PsA patients with arthritis and in synovial fluid of established PsA patients with active disease.

Methods: Fresh blood was taken from first diagnosed DMARD and steroid naïve PsA patients $(n=10)$, having arthritis in 1 or more joints (PsA blood). The diagnosis was made by a rheumatologist according to the CASPAR-criteria. In addition, fresh synovial fluid was obtained from established PsA patients (PsA SF) with active disease $(n=10)$ and treated with either methotrexate $(n=3)$ or adalumimab $(n=3)$ or NSAIDs $(n=4)$. Multicolor flow cytometric analysis was performed on PsA blood and PSA SF. For the detection of IL-17A, IL-17RA or IL-17RC the following antibodies were used: IL-17A-PE (eBioscience), IL-17RA or isotype control IgG1k (both Biolegend), IL-17RC or isotype control IgG2b (both R\&D systems). The following markers were used to discriminate between different cell populations: $T$ cell subsets (CD3, CD4, CD8, CD45RO, CCR6, TCR $\gamma \delta$ ), B cells (CD19), NK cells (CD15-CD16+), neutrophils (CD15+CD16+), monocytes (CD33+CD14+CD16+/-), mast cells (CD117+FcER1a+) and eosinophils (CD15+FcER1a+).

Results: Different lymphoid and myeloid cell types were IL-17A positive in PsA blood of first diagnosed PsA patients such as CD3+, TCR $\gamma \delta+$, CD4+, CD8+ lymphoid cells, CD14+ monocytes and eosinophils. In PSA SF of established PsA patients TCR $\gamma \delta+$ T cells, neutrophils, NK cells and eosinophils were IL-17A positive.

In both groups, no difference in expression of IL-17RA and IL-17RC was found on CD4+, CD8+, CD4+CD45RO+CCR6+/-, TCR $\gamma \delta+$ and CD19+ lymphoid cells compared to their isotype control. In contrast, the expression of IL-17RA and IL-17RC was increased compared to their isotype control on neutrophils and monocytes in PsA blood and on neutrophils, monocytes, mast cells and eosinophils in PSA SF.

Conclusions: These preliminary data show that not only lymphoid cells but also specific myeloid cell types may be sources of IL-17A in PsA. Furthermore, not lymphoid cells but IL-17RA/IL-17RC positive myeloid cells such as monocytes, neutrophils, mast cells and eosinophils may be potential target cells for IL-17A

Together, these data suggest a more broad, but specific IL-17A-IL-17RA/RC signaling network between different cell types important in the IL-17A-driven pathogenesis of PsA.

References:

[1] Lubberts E. Nat Rev Rheumatol 2015, 11: 415-29.

[2] McInnes IB, et al. Lancet 2015, 386: 1137-46.

Disclosure of Interest: None declared

DOI: 10.1136/annrheumdis-2017-eular.5916

\section{AB0034 PARAMETERS OF TOTAL BLOOD COUNT; MIGHT THEY BE INDICATORS OF INFLAMMATION IN RHEUMATOID ARTHRITIS AND ANKYLOSING SPONDYLITIS?}

O.G. Illeez, F. Unlu Ozkan, I. Aktas. PMR, University of Saglık Bilimleri, Fatih Sultan Mehmet Training and Research Hospital, Istanbul, Turkey

Background: Neutrophil to lymphocyte ratio (NLR) and platelet to lymphocyte ratio (PLR) are launched as recent markers of inflammation in chronic inflammation principally in cancer and cardiovascular diseases (1-2).

Objectives: Rheumatoid arthritis (RA) and ankylosing spondylitis (AS) are chronic inflammatory disorders marked by variable periods of remissions and relapses.Inflammation is most likely the underlying cause in disability, increased comorbidity therefore need to be closely monitored and kept under control (3).For this reason cost effective, accessible and reliable parameters are needed in daily practice. Our aim is to analyze the relation between inflammation and NLR and PLR which are easily calculated from whole blood count parameters.

Methods: Medical records of 425 subjects were analyzed retrospectively.Mean age of the subjects was $44,64 \pm 14,07$ years (17-89 years), $52.9 \%$ was female $(n=225)$ and $47.1 \%$ was male $(n=200) .105$ of them had RA, 216 of them had AS and 104 were healthy controls.2010 ACR/EULAR classification criteria and modified New York criteria were used for the diagnosis of RA and AS.Erythrocyte sedimentation rate (ESR), C reactive protein (CRP) and whole blood count were recorded with simultaneous DAS28 scores of patients with RA and BASDAI scores of patients with AS.

Results: Hemoglobin levels of RA patients were significantly $(p<0.05)$ lower then the levels of control group $(p=0.001)$.ESR, CRP, NLR and PLR were significantly higher then the control group respectively $(p=0.001, p=0.001$, $p=0.001, p=0.040$ ). In AS group hemoglobin, ESR, CRP and NLR values were significantly higher then the control group respectively $(p=0.001, p=0.006$, $p=0.001, p=0.001$ ). No difference was detected between AS and control groups in terms of PLR $(p>0.05)$. When patients with high disease activity and patients in remission were compared for both $R A$ and AS groups ESR $(p=0.001, p=0.001)$ 